\title{
An AHP based Group Decision Framework for Dynamic Location Selection in Global Manufacturing
}

\author{
Mitchell M. Tseng, Xiaoke Zhang "* and Xianyun Wang \\ Department of Industrial Engineering and Engineering Management \\ Hong Kong University of Science and Technology \\ Clear Water Bay, Hong Kong \\ tseng@ust.hk/iezxk@ust.hk/iewxy@ust.hk
}

\begin{abstract}
In today's fierce competitive business environment, keeping some competitive advantages is crucial for a manufacturer to survive and prosper. Globalization is an important way for attaining such advantages. The success of global manufacturing is highly related to many strategic decisions. Location selection is one of those unavoidable decisions. A good location selection may help a firm to achieve lower manufacturing cost and better customer service; while a bad one may add extra costs to a firm and it may even damage a firm.

Location selection for global manufacturing can be extremely difficult due to the uncertainty and volatility of international environments. In addition, many factors influencing the final decision may conflict with each other. For example, the lower labor cost in Asia can be undermined by strict quota limitations in textile industry. More than that, strategic decisions such as the location selection, usually involve a group of decision-makers. Concerning continuous improvements or variation in the world and regional economic and political environments, an attractive area or country over one period may loss its charm to investments in another period. For a long-run concern, the dynamic aspect or the multi-period perspective of location selection can not be neglected.
\end{abstract}

Therefore, effective location selection should simultaneously address the following four aspects:

a) Multiple possibly conflicting criteria including tangible and intangible ones

b) Incomplete and fuzzy information

c) A group of decision-makers involved in the decision process, and

d) Multi-period perspective

The Analytic Hierarchy Process (AHP), being one of the most influencing decision support methods, has been successfully applied to explore facility locations.

The early application of AHP in location selection can be found in J. A. Wu and N. Wu's work (1984). They employed AHP to select the best single plant location for a hypothetical furniture company. A two-level hierarchy, with both tangible and intangible factors, was considered. Using AHP as the engine, Yurimoto and Masui (1994) designed a decision support system for overseas plant location selection. A number of qualitative factors such as contributions to the host country and its social environment were considered in the AHP model. This system was applied to Japanese affiliates in the EC and was proved its validity. Other applications of AHP in location selection can be found in Nezhad and Kathawala (1987), Banai-Kashani (1990), Declercq and Peeters (1994), Min (1994), Tofigh.(1996), and Yang etc. (1997)

Although there are a bundle of applications of AHP in location selection, the characteristic of the group decision making and the dynamic aspect of the location selection in global manufacturing have been overlooked. Consequently, an AHP-based framework for group decision in dynamic location selection of global textile manufacturing is proposed in this paper. Within this framework, a four-level hierarchy is constructed. As described in the following figure, the decision criteria are listed in the second level; in the third level, each criterion is decomposed into several sub-criteria; the fourth level represents location alternatives to be selected.

" corresponding author 


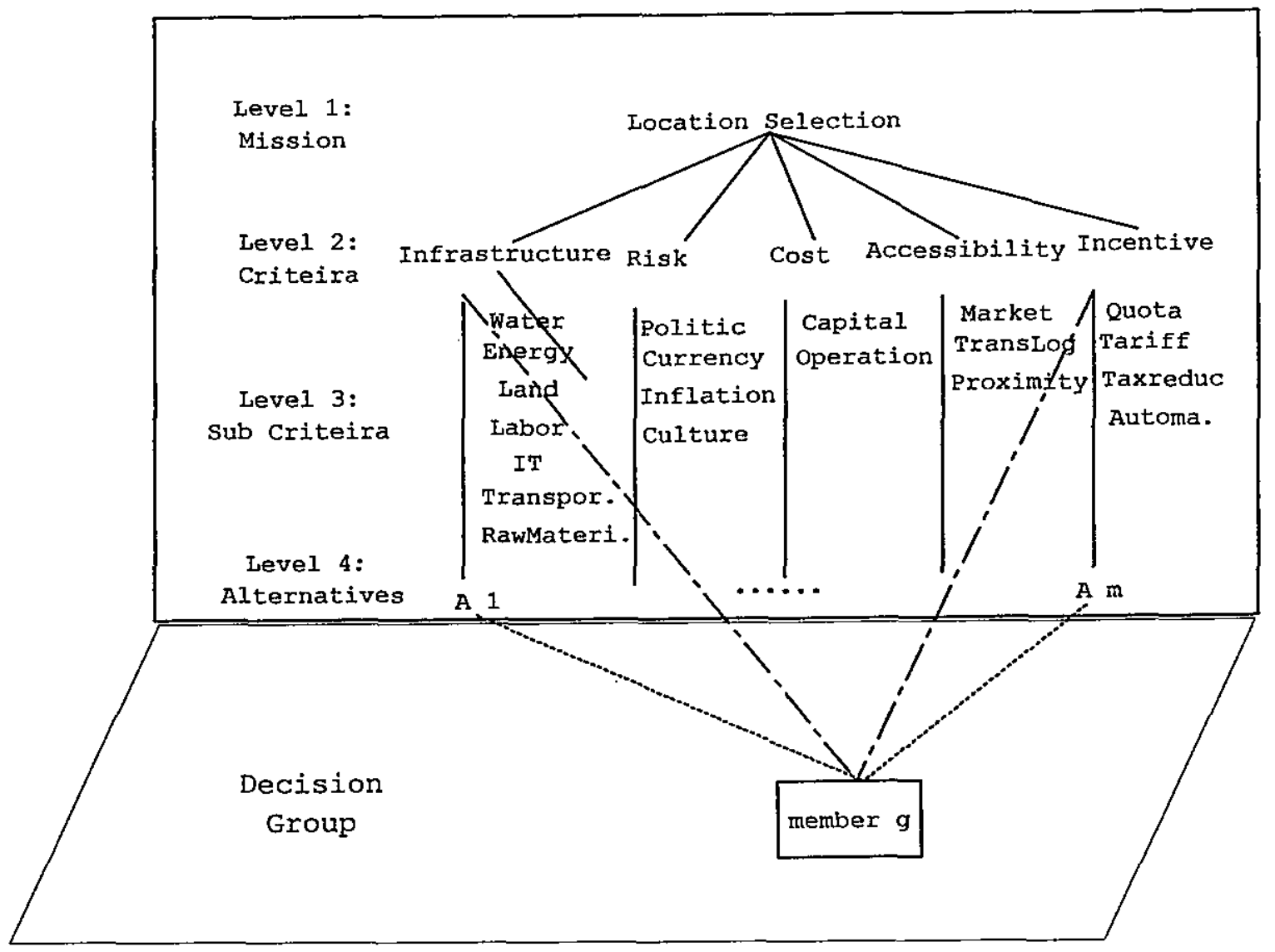

The group members construct the hierarchy together, then the priority of each criterion is set by pairwise comparison. If group consensus can not be reached, different weights are assigned to the group members according to their expertise. For example, on the second level, the CEO has the highest weight when determine the priorities of criteria; In the lower level, IT experts will have the highest weight when decide the priority of IT as a sub criterion of infrastructure, while economist might has higher weight on currency of risk assessment. The weight of a group member is not fixed. By this approach, the group decision making is more close to the real situation compared with using geometric mean or assigning a fixed weight to a group member without considering the diversity of problem domain and domain of expertise.

Taking the dynamic aspect into consideration, it is natural to add time dimension into the framework. The hierarchy may be the same, but the priority of each element varies corresponding to different time period. Take location alternative A and B as an example. Place A will attract enough investment in several years, then the labor cost will increase and other resource capacity will be a limitation; at the mean time, place B will improve their incentives by signing free trade agreement with other countries and may have more resources compared with $\mathrm{A}$. As a result of time, the priorities of criteria will vary as well as preferences of alternatives shift.

The uniqueness of this framework is as follows: 1) the capability of adjusting the weights of group members across the hierarchy, i.e., one member may have different weights when different criteria are discussed; 2)the dynamic aspect of the location selection is considered. With a given time scope, a rational decision can be made. This framework provides communication, computing and decision support capabilities to aid group idea generating, planing, problem solving and decision making. In addition, it facilitates decision makers to evaluate and explain their decisions from different viewpoints. It also offers a flexible mechanism to carry out sensitivity analysis.

Keywords: AHP, group decision, dynamic location selection, global manufacturing 